\title{
Blockchain Governance: What We Can Learn from the Economics of Corporate Governance
}

Darcy W. E. Allen and Chris Berg

RMIT Blockchain Innovation Hub, RMIT University, Australia

Correspondence: darcy.allen@rmit.edu.au

Received: 20 February 2020 Accepted: 16 March 2020 Published: 30 March 2020

\begin{abstract}
Understanding the complexities of blockchain governance is urgent. The aim of this paper is to draw on other theories of governance to provide insight into the design of blockchain governance mechanisms. We define blockchain governance as the process by which stakeholders (those who are affected by and can affect the network) exercise bargaining powers over the network. Major considerations include the definition of stakeholders, how the consensus mechanism distributes endogenous bargaining power between those stakeholders, the interaction of exogenous governance mechanisms and institutional frameworks, and the needs for bootstrapping networks. We propose that on-chain governance models can only be partially utilised because of the existence of implicit contracts that embed expectations of return among diverse stakeholders.
\end{abstract}

Keywords: Blockchain Governance, Institutional Cryptoeconomics, Economics of Blockechain, Corporate Governance

\section{Introduction}

Blockchains are decentralised digital network protocols whose governance is characterised by a complex interplay between stakeholders. An incomplete list of these stakeholders includes token holders, network validators (such as Bitcoin's miners and economic full nodes), core and application developers, and founders. Each of these stakeholder groups have a stake in the protocol and each face sharply different incentives when considering whether and how the protocol should be modified. Many other stakeholders don't actively participate in the network but have interests in its structure and modification. These groups include government regulators, activists, media and social media, participants in competing and complementary blockchains, and other parts of the technology stack. The blockchain governance challenge is how to design and build systems that balance the interests of each of these stakeholders and ensure the success of the network, however that success is defined [1].

Social organisations such as corporate firms are made up of individuals that have diverse ends seeking to make exchanges and modify or sustain the environment in which they make those exchanges. They seek to make decisions, implement those decisions, and monitor their implementation and outcomes. These decisions are necessary because not all future states of the world can be identified at the exact moment of organisational formation. Organisations need to adapt to survive [2]. Governance describes the processes by which individuals and groups with ongoing relationships bargain about how to adapt to changes within an institutional environment-such as a firm, a political or community organisation, or in market contracting [3-6].

Whether the institutions of governance have been designed explicitly or not, all blockchains have governance. While those governance systems vary in their effectiveness [7], governance itself is a descriptive, rather than a normative, attribute. Blockchains can be thought of as competing constitutional rule sets, where they compete on rules for making rules [8]. In this way, blockchain governance relates to the way decisions are made, not the decisions themselves - who chooses and how choices are made, rather than what is chosen $[9,10]$.

Since the whitepaper by Nakamoto [11], which groups should be considered stakeholders in blockchain governance-as well as the formal and informal structures for decision-makinghas been highly contested. Owing to their past decisionssuch as investing in tokens in the early stages of a networkbargaining power is asymmetric between stakeholders. Stakeholders, and the groups they form, face distinct set of costs in past and future investments in the network-that is, they have made asset-specific investments that constrain future decision-making. On public blockchains such as Bitcoin that allow for open entry and exit (holding and transacting coins, as well as observing the chain and validating new transactions), bargaining power is relevant when modifications to the underlying protocol or core software are proposed. Initial decisions about the governance of the Bitcoin protocol 
were made by the founder(s) directly or in consultation with a small online community. Later, decisions about protocol modifications to facilitate network scaling were subject to intense bargaining between stakeholder groups-dominated by miners - and led to some uncertainty about the future of the network [a detailed exploration of this scaling debate is provided by 12]. In a user-activated soft fork in 2017, token holders and economic nodes demonstrated that non-mining stakeholders could also exercise bargaining power within Bitcoin's governance structure.

Governance disputes surrounding blockchain protocols have extended beyond Bitcoin (including, for instance, the DAO hack on Ethereum) and have raised important questions on how blockchains should be governed. It is common to distinguish between "on-chain" or "off-chain" governance [see 13, 14, 15]. On-chain governance describes the project of explicitly building governance arrangements within the protocol itself, such as the implementations of EOS, Tezos, and Dash that allow certain categories of stakeholders to vote on modification proposals. Off-chain governance typically describes governance structures external to the protocol, particularly the role and management of foundations or firms funded by token sales or other token distributions (for example, Zcash's Electric Coin Company and Zcash Foundation), or community meeting places such as Reddit, Telegram, Slack, dedicated forums, and Twitter.

In this paper we aim to provide a descriptive framework for understanding blockchain governance, from which we draw some normative implications. Our goal is to contribute to a deeper understanding that blockchain entrepreneurs can draw from when designing governance systems. "Blockchain" is a generic term for a prominent subclass of distributed ledger technologies, "multi-party systems that operate in an environment with no central operator or authority, despite parties who may be unreliable or malicious"; see Rauchs et al. [16]. We limit our investigation to public blockchains, rather than permissioned or private blockchains (while recognising that the difference between the two is not clear at the margin). While our insights have relevance to permissioned blockchains (including, for instance, defining who should be permissioning), there are some clear differences particularly relating to the definition of stakeholders (and thus control) in permissioned networks, the different needs of bootstrapping, and the problem of forming and governing consortia. We leave these important questions to future research.

We draw on a coherent body of theory around institutional economics - a body of thought structured around the governance of contractual relationships. This body of thought, including transaction cost economics, brings together economics, law, and organisation theory to make the transaction as the basic unit of analysis and includes contributions by Ronald Coase (on why firms exit), James Buchanan (on club goods and constitutional rules), Oliver Williamson (on the economic institutions of capitalism), Oliver Hart (on incomplete contracting and make-or-buy decisions), and Elinor Ostrom (on commons) [17-25]. This coherent body of thought has been applied specifically to blockchain networks through institutional cryptoeconomics [26-30].

While much of the institutional cryptoeconomics has focussed on the effect of blockchain as an institutional technology, in this paper we focus on what institutional economics can teach us about the governance of blockchains themselves. We explore several questions regarding blockchain governance. Who is a stakeholder in blockchain governance? To what extent are blockchain governance systems unique? How can effective long-term blockchain governance be consistent with the needs of bootstrapping-the process of building a blockchain network from the ground up [28]?

We start from corporate governance rather than network governance [31], technology ecosystem governance [32], or nodal governance [33] for two reasons. First, blockchains have algorithmically specified structures that deterministically distribute bargaining power within the network. While this distribution is not hierarchical, as in a firm, neither does it meet traditional understanding of informal reciprocal and social network governance, in that a blockchain network is a domain of formal (smart) contractual exchange. (We discuss the differences between blockchain governance and another network governance-the internet-in Section 5.) Second, attempts by the blockchain industry to design formal on-chain governance systems suggest to us that it is most valuable for researchers to start with the formal governance of the corporation and work their way out from there. At the first instance, a blockchain is a platform for $n$-sided market contractual exchange [34], around which a technology ecosystem is built.

This paper makes several contributions to the literature on blockchain governance. Blockchain governance is the process by which stakeholders - all those that are affected by and can affect the network-exercise bargaining power over the network itself. This includes token holders, miners, and founders. But blockchains interact with, and are shaped by, external institutional frameworks, such as the firms that act as institutional investors for tokens or other organisations up and down the stack, the firms that provide exchange services, and government regulators who impose requirements (such as know-your-customer and anti-money laundering regulations) on the on-ramps to the network. We draw on the literature on corporate governance with a focus on implicit and explicit contracts, and how management and governance deals with those complexities.

We introduce three distinct elements that shape our understanding of blockchain governance: endogenous governance, exogenous governance, and the need for bootstrapping. We offer a new distinction between the distribution of bargaining power endogenous to the consensus mechanism and the exogenous governance structures that are built on top. Endogenous governance describes the bargaining power that is directly derived from instrumental features of the consensus mechanism. That is, 
elements of the protocol that are minimally necessary for achieving consensus. In a proof-of-work protocol like Bitcoin, bargaining power is determined by the instrumental roles of miners and full economic nodes. Endogenous governance is the distribution of power between stakeholders directly involved in the consensus mechanism. We also discuss the needs of bootstrapping in the early stages of the network and how this affects the distribution of bargaining power. These elements provide, sometimes, contradictory pressures towards and against decentralisation.

\section{Who is a stakeholder in blockchain governance?}

Blockchain governance faces a boundary problem. Before we can determine how a governance mechanism is structured, we need to define the boundaries of who is doing the governing-that is, who are the stakeholders. Whether a blockchain governance system is implicit (that is, the allocation of bargaining power comes from the instrumental design of the consensus mechanism) or planned (where the protocol has been designed specifically with a governance mechanism in mind), we need some guidance about which stakeholders are analytically relevant for the assessment and design of governance mechanisms.

We can look to our understanding of how we define corporations and their governance-from the responsibility to deliver profit to broader conceptions of corporate social responsibility - to understand stakeholders in blockchains. Milton Friedman [35] famously wrote that the sole responsibility of a firm is to generate profits to its shareholders. A formal model of this describes the firm as a nexus of contracts between investors, managers, and their subordinates, where the residual income accrues to shareholders [25, 36]. Thus, corporate governance under this framework describes the process by which shareholders ensure that profits for their investments are returned-that managers do not abscond with money already invested [37].

The transaction cost approach to the firm emphasises how the inevitably incomplete contracts that make up firms shape institutional choices [21, 22]. This tradition of framework can be described as a contracting-first approach to corporate governance, and pivots around the ownership and use of property rights in the organisation.

The corporate social responsibility movement has challenged the shareholder-first framework [38], arguing that a wider variety of groups and interests should be taken into consideration by the management. Rather than being simply responsible towards shareholders, firms ought to be responsible towards "stakeholders." In many ways, corporate social responsibility is the process of stakeholder management [39]. But as Janita Vos [40] asks, who is a stakeholder? A stakeholder can be any group or individual who can affect the governance or an operation of an organisation or is affected by it $[41,42]$. The first true list of stakeholders for the management was that attributed to the vision of General Electric in 1931: shareholders, employees, customers, and the general public [43, 44]. But other groups could, of course, be affected, or could affect the organisation.

Governance discussions around blockchains have typically narrowly defined the categories of a stakeholder, either implicitly or explicitly. Disputes around the scaling of Bitcoin, for instance, have sometimes been seen as a narrow bilateral dispute between Bitcoin core developers - those who work on the reference implementation of the Bitcoin software-and miners who validate the chain and compete to mint new tokens $[45,46]$. Explicitly designed governance mechanisms are likewise constrained. EOS and Tezos, two blockchain protocols with such explicit governance, give token holders voting rights over delegated validators and modification proposals, respectively. But token holders are not the only potential stakeholders who might be affected (and can affect) governance decisions. It is worth noting that in EOS and Tezos, validators and core developers are not explicitly identified as stakeholders for formal on-chain governance except insofar they may also hold tokens (whether to maintain a stake in the system or as part of receiving and disposing of block rewards). In this way, different stakeholder groups may be highly correlated.

We can identify a wide range of separate stakeholder groups. Even in Bitcoin, token holders are not a homogenous group. Governance analysis might distinguish between token holders who intend to use their holdings primarily as a medium of exchange, and those who are holding them as speculative assets (HODLers). Founders and founding foundations can affect the decisions of the protocol. Developers can be divided into core developers (with or without repo access) and developers who are building applications that use the protocol as an infrastructure layer. Economic full nodes, such as large, over-the-counter traders, token holders, and miners can have distinct stakeholder interests. The producers of hardware that support the chain (ASIC or GPU producers, cold storage wallets, etc.) are also stakeholders.

We can expand the stakeholder groups further when considering individuals or groups who are affected by the blockchain but do not directly interact with it. Bitcoin provides a medium of exchange and unit of account for holders of other cryptocurrency tokens. A typical exchange denominates cryptocurrency in units of Bitcoin. Initial coin offerings on EC20 tokens typically involve an initial acquisition of the Ethereum token, Ether. Bitcoin and Ethereum stakeholders can be said to have the power to affect holders of other cryptocurrencies. In this sense, the financial system itself can affect cryptocurrencies through its interaction with blockchain on-ramps (exchanges, payment networks, etc.) or through competition or even simply through the price level. Furthermore, industries which are disrupted by specific blockchain (supply chains, logistics, data science, health, etc.) might also be said to be stakeholders. Government authorities that have regulatory responsibility for fields in which blockchain applications operate are also stakeholders. In some circumstances, social groups can be described as stakeholders. The significant electricity use of proof-of-work consensus mechanisms, and its potential impact on the global energy use, 
means that environmentalists, of their representative nongovernment organisations, could be stakeholders, insofar as they are affected by the operation of the protocol.

Given the potentially wide range of stakeholders, and the complexities in identifying them, which groups should be considered stakeholders while maintaining workable governance structures? Too many stakeholders exercising control rights over an organisation can privilege the interests of groups with little stake above those who are most directly affected, or alternatively, where delegation has been given to authorities to weigh interests, allowing managers to hide selfinterested behaviour [47]. Responding to this challenge, the corporate social responsibility tradition has sought to distinguish between different groups of stakeholders. Fassin [48] and Fassin [49] propose a division between "real" stakeholders, whose influence over the firm is the organisation (insofar as they have control rights over the organisation, the organisation has control rights over them), "stakewatchers," who represent the interests of real stakeholders (such as unions, consumer groups, environmental groups, and investor associations), and "statekeepers," who have no stake in the firm but impose constraints (such as government agencies and regulators).

By contrast, the contract approach structures its answer to who is a stakeholder around property rights as residual rights to income [36] or residual control rights [50]. In this approach, stakeholders are "all investors who create transaction- and/or firm-specific property under the reasonable expectation of a return on investment through interaction with the firm" [51]. Here, the legitimate group of stakeholders encompasses those who have both explicit and implicit contracts with the firm. Explicit contracts are those contracts not directly stated but understood by both parties for the contract to exist. Implicit contracts recognise the existence of the co-creation of value and the expectation of a real return for such investments. Such informal quid pro quos are pervasive within the firm, and even explicit contracts are hard to navigate without some understanding of the implicit agreements that underpin them $[52,53]$. Incorporating this understanding of implicit contracts for the co-production of economic value considerably narrows the otherwise infinite space of stakeholders. Implicit contracts are contracts which are obscure to outside observers. Indeed, because implicit contracts are not written down and are based in norm rather than a clear agreement, they obscure the ultimate economic value of an organisation and the search for general principles that might apply across organisations. In a firm, some "outputs" — such as the training of employees — are neither priced nor explicitly documented.

Implicit contracts exist in blockchain networks, most obviously through the roles played by founders, foundations, and developers. But in a firm, it is the job of the management to weigh and balance the implicit contracts [54, 55]. Stakeholders can implore the management to weigh their interests more heavily, and penalise the firm through (a) reputation loss and (b) a choice not to make further investments if they are not satisfied. Firm managers have the discretion to distribute income to stakeholder groups, identifying and responding to implicit contracts as necessary. They are constrained from doing so in their interests to the extent that the explicit contracts with shareholders prevent such opportunistic behaviour [47]. By design, decentralised organisations have no "management." No single class of stakeholder is empowered to coordinate implicit contracts. This obviously protects against a category of rent-seeking behaviour caused by agency losses between the owners and the management. But it leaves uncertainty as to how the distribution of value-derived implicit contracts can be negotiated between stakeholders.

\section{Endogenous and exogenous governance}

In this section, we examine the mechanisms through which governance decisions over implicit and explicit contracts are made. We distinguish between two forms of governance of blockchain networks: endogenous and exogenous. Blockchains have endogenous governance systems that create the relative bargaining power instrumentally determined by the consensus mechanism. We argue that the initial design of a blockchain consensus protocol maps to a different distribution of bargaining power over the network itself. Furthermore, blockchains also have exogenous governance systems that are the formal and informal governance processes that exist outside of the instrumental needs of distributed consensus over the state of the ledger. Our analysis is distinct from the endogenous-exogenous split presented by de Filippi and Mcmullen [56] because it pivots on whether governance is determined by the consensus mechanism, which Rauchs et al. [16] describe as the characteristic that makes distributed ledger systems unique.

The distribution of bargaining power over blockchain governance, at the first instance, is endogenously governed by the consensus mechanism. Bitcoin is a three-sided market between miners, buyers, and sellers [34]. The dominant players are economic full nodes-those who keep a complete copy of the chain, broadcast transactions, and validate the shared ledger $[57,58]$. Their decision of whether to adopt software amendments produced by core developers depends on whether they believe that other economic full nodes will accept new blocks produced by the software. In other words, it is the economic full nodes that enforce the rules. Their ability to accept or reject blocks following different rules gives them endogenous bargaining power and, therefore, the governance control over the network. How precisely this distribution manifests itself in decisions depends, of course, on the interests of the economic full nodes as individual agents, but structurally the consensus mechanism gives them the governing power over the network.

Our focus here is not on whether blockchains have designed or not designed governance processes, or between blockchains with or without governance. Rather, we emphasise that the structure of the consensus protocol determines the bargaining power. Stakeholders in endogenous governance have been given formal bargaining power over the network by the design of the consensus protocol. A parallel here is with the formal institutions of a firm-shareholders, management, and 
employees - that forms the "machine" for profit-seeking economic activity. Endogenous governance can be intentionally designed. While Bitcoin was built without governance in mind, in EOS a "governance" system has been built into the consensus mechanism which allows token holders to vote for block validators. This produces an alternative distribution of bargaining power, where token holders (and their proxies) exercise a significant amount of power (relative to the Bitcoin network).

By contrast, exogenous governance describes the formal and informal governance processes that exist outside the instrumental needs of distributed consensus over the state of the ledger. These can be formally designed or evolved in response to a perceived need for legitimacy. Exogenous governance can be "on-chain" or "off-chain", "formal" or "informal," as described by Buterin [59], Buterin [60], Zamfir [61], and Ehrsam [62]. At Coindesk's Consensus conference in 2017, an agreement (the "New York Agreement") was brokered between 56 separate mining and Bitcoin application firms for two modifications of the protocol: segregated witness and larger block sizes [63]. The exogenous governance mechanism here is provided by the opportunity for coordination presented by the Consensus conference itself. De Filippi and Loveluck [1] describe this process as the "invisible politics" of Bitcoin. In the wake of the hack of The DAO, a voting mechanism was created to vote on whether to hard fork Ethereum to reverse the hack. The hard fork was triggered in July 2016. On-chain mechanisms for voting on protocol modifications (such as the ones offered in EOS, Tezos, and Dash) are exogenous insofar as they do not form an instrumental part of the consensus function.

Endogenous and exogenous governance mechanisms co-exist, providing mutual restraints against each other. Where governance has been explicitly designed, it is still subject to endogenous governance processes. The creation of Ethereum Classic after The DAO hard fork underlines the persistence of endogenous bargaining power after the creation of exogenous governance, albeit with the result being a split in the network. In EOS, the delegated proof-of-stake consensus mechanism allows token holders to vote for validators (block producers), and also to vote on decisions about the protocol (referendum proposals). The distribution of bargaining power determined by the former voting system is endogenous and the latter exogenous. Both endogenous and exogenous governance processes are subject to evolutionary pressure as technical developments (such as ASICs) and entrepreneurial innovation (such as mining pools) reshape the relative bargaining power of stakeholder groups [8,64].

We can see here how the co-existence of implicit contracts between diverse stakeholders and blockchain governance systems creates challenges. Implicit contracts in decentralised systems have to be constantly negotiated, in the same way that corporate culture as a tool for the negotiation of implicit contracts is subject to constant evolution and evaluation. Particular on-chain exogenous governance systems that provide a formal mechanism for token holders (weighted by token holdings) to vote on protocol-level changes elide these complex multi-party negotiations by identifying a singular distinct category of stakeholders whose preferences are most convenient to collate.

\section{Governance and the needs of bootstrapping}

The distribution of bargaining power of endogenous governance is set instrumentally by the consensus mechanism. The domains of exogenous governance, on the other hand, is more diverse. Exogenous governance can be built into the protocol as a referendum process (as in EOS and Tezos) or revolve around over the norms and cultural structures of the community of users. Those norms and cultures vary significantly [65] and determine whether exogenous governance decisions are seen as legitimate by all stakeholders.

One obvious illustration of the role of legitimacy around exogenous governance norms is the governance role of Satoshi Nakamoto in the early days of Bitcoin, and the subsequent function played by the Bitcoin Foundation. Satoshi's "vision," as outlined in the Bitcoin whitepaper [11] and subsequent mailing list and forum posts, has played an outsized role in shaping governance choices over the network. Likewise, core developers have a governance role that does not simply reflect their instrumental function within the consensus mechanism. In parallel to democratic governance, some exogenous governance mechanisms rely on leaders and key players to provide guidance and heuristics for people to make decisions about blockchain governance. Some relatively informal exogenous governance mechanisms - such as leaders and early adopters-might ameliorate the costs of making more formal governance decisions.

A fruitful but extensive task would be to audit blockchain communities looking for commonalities in these norms and cultures [one early attempt to do so is 10]. Here, however, we start from the question: how do those norms evolve? There is at least one consistent feature of all blockchain networks. They must start from somewhere. They must all be bootstrapped.

Blockchain protocols are the result of entrepreneurial creative discovery [66]. They come from specific environments-from the mind of entrepreneurs and their relationship with other idea producers. In this Kirznerian tradition, Allen [67] and Potts [68] explore how ideas are governed as they are combined and recombed in the proto-entrepreneurial stage. To bring ideas to market, organisational structures are created so that the property rights over those ideas can be allocated [27, 69]. The organisational creation need not be a firm. It can be as simple as writing a white paper that describes the protocol for a new business model, marking that code as open source, and posting it on a websit. Alternatively, many blockchain networks have undertaken initial coin offerings that have raised substantial funds for development and to subsidise development work within their communities [29, 70-72]. For blockchain networks, these two stages - the proto-entrepreneurial and the organisational-leave their mark on the later governance and shape the distribution of bargaining power by later stakeholders. 
Blockchains are not born decentralised. Catalini and Gans [28] describe Bitcoin as the first digital platform to be bootstrapped without the need for investment from a planner or other intermediaries. But bootstrapping still requires work. Whether Satoshi was an individual or group of individuals, specific individuals had to design the software and write the Bitcoin white paper. New innovations need hype to facilitate earlystage cooperation, and the hype is an economic good that has to be produced [73]. Even if Bitcoin emerged fully formed from the mind of a single "Satoshi Nakamoto," in the early stages of Bitcoin, decisions as to the design of the protocol were negotiated between different stakeholders through Bitcoin talk forums, newsgroups, and email lists. One prominent picture of the governance of Bitcoin around the needs of bootstrapping is the December 2010 debate of whether Wikileaks should be encouraged to use Bitcoin for donations, which was at the time resolved in favour of an appeal from Satoshi to Wikileaks not to adopt the fledgling cryptocurrency $[74,75]$.

The process of bootstrapping exerts an influence on the norms around governance and the implicit contracts that are negotiated long after an initial bootstrapping phase. For example, De Filippi and Loveluck [1] describe a belief implicit in Bitcoin governance processes that "the Bitcoin core developers (together with a small number of technical experts) are-by virtue of their technical expertise- the most likely to come up with the right decision as to the specific set of technical features that should be implemented in the platform." Recent work on the economics of corporate culture underpins the role that culture plays in coordinating expectations between the management and the employees who have made specific investments in the firm [76-78]. We can understand these relationships within the network as subject to implicit contracts that enhance the network's economic value.

These implicit contracts have a clear origin-the entrepreneurial creation of the protocol and the need for bootstrapping a network-but by their nature are hard to be pinned down with any formality and are highly contextual. They explain the role played by Satoshi in Bitcoin's early days, and the shifts in governance since Satoshi's disappearance. Disputes over the Satoshi legacy and the increasing contestability of the role of the Bitcoin core developers are a form of renegotiation of this implicit contract. Satoshi's absence from the Bitcoin community since December 2010 is an unusual case. Founders and their founding organisations play a key role in the creation and bootstrapping processes. Their structural roles (for instance, as core developers or block validators) and the implicit contracts that have been built around them tend to be controversial. Examples include the role of Vitalik Buterin and the Ethereum Foundation, the position of Block. One as the developers of the EOS network, and the Zcash founders' reward. These founders and organisations do not have any endogenous role in blockchain governance as determined by the consensus mechanism. But their role as exogenously determined stakeholders and the implicit contracts that support that role create a dilemma for blockchain governance, given political beliefs about decentralisation within many blockchain communities.

\section{The ends of blockchain governance}

Corporations are treated in law as intentional systems [79, 80] - that is, corporations are an entity, even a moral entity, in and of themselves. Alchian and Demsetz [36] argue that firms are units of team production, where the possibility of teamwork is limited by the costs of disciplining/shirking - that is, corporations are the aggregation of a nexus of contracts [81]. These views are typically seen as contrasting [82] but each imputes to the corporation a particular-if not quite a singular-purpose. While each contractor to the firm (employee, management, and shareholder) seeks their own ends, the team is organised in the pursuit of a singular end. The governance of a firm consists in coordinating around that singular end, whether it is profit-maximisation in the Friedman sense or ends determined by an assessment of the corporation's social responsibilities.

So, what are the ends of blockchain governance? An implicit end common to the blockchain community is that the network survives-that is, it maintains its immutability through distributed consensus while accepting new transactions-and is adopted more widely. The Bitcoin governance crisis described by De Filippi and Loveluck [1] concerned these two ends. Of course, the ends of different categories of stakeholder groups are heterogeneous between and within those categories. Token holders who hold tokens as an investment might want the value of their holdings to increase relative to fiat currency, while application developers who wish to use tokens as a utility in their applications often want price stability. While each stakeholder group shares a distributed network, they pursue different final ends.

These stakeholders use blockchain as a shared economic resource with which they pursue different ends-that is, blockchain is an infrastructure [83]. Frischmann [84] offers a set of characteristics that make a resource infrastructural: its consumption is non-rivalrous within certain demand bounds, its demand is a function of downstream production, and it is an input into a wide array of goods and services. A given blockchain offers generic public capabilities that allow for diverse productive ends to be pursued. The shared interest is in the maintenance of that infrastructure and its increased utility of the infrastructure, which exploits possible network effects.

We might compare blockchain governance then with internet governance, another shared digital infrastructure. The United Nations Working Group on Internet Governance [85] describes governance as "the development and application by governments, the private sector and civil society, in their respective roles, of shared principles, norms, rules, decisionmaking procedures, and programs that shape the evolution and use of the Internet." Nonetheless, as Van Eeten and Mueller [86] note, debates over internet governance have tended to focus disproportionately on a small number of formal institutions and quasi-government stakeholders (such as the Internet Corporation for Assigned Names and 
Numbers) while downplaying the role of (for instance) internet service providers, telecommunications regulators, operating system developers, and mobile phone device manufacturers who fit within that definition.

Likewise, using the expansive approach to stakeholder identification described in Section 2, the active participants in exogenous blockchain governance include stakeholders all the way through the stack, from the telecommunications providers who host the distributed network, the GPU and ASIC manufacturers who produce mining equipment, application developers, chains launching their native tokens on other chains, venture capital firms investing in application developments, to government standard bodies and financial sector regulatory agencies. Importantly, these governance stakeholders do not all share the same ends-not all of them have the shared interest in the maintenance and increased utility of the blockchain-yet all can exercise a degree of control about the future of the blockchain network. The parallel between internet governance and blockchain governance should encourage researchers to cast their net wide for stakeholder identification.

Nonetheless, the differences between internet governance and blockchain governance are substantial and relevant. Putting aside possible balkanisation of the internet [87], the internet is a singular shared protocol. By contrast, there are many competing blockchain protocols. They compete on different margins and evolve and fork at different speeds. Furthermore, the use of one blockchain does not preclude the use of others, partly because they each operate on internet infrastructure.

A more fundamental difference between the internet and blockchain governance is the role that blockchain tokens play in coordinating maintenance of the network. Tokens align incentives by endogenising the capital formation necessary for bootstrapping [28]. While the internet has a variety of institutional governance frameworks-such as corporate, government, and commons [84]-blockchains can be understood as a self-contained institutional technology [27]. Yet the managers of corporations are constrained by fiduciary duties specified in law that require them to act both in the interest of shareholders and the company. As an institutional innovation, stakeholders in blockchains lack these legal constraints. Neither token holders, miners, nor full economic nodes are required to act in others' interests. To the extent that they do, it is because the consensus mechanism and native token coordinate self-interested behaviour to maintain and protect the network.

\section{Conclusion}

In this paper we have drawn on the theory of corporate governance to better understand the complexities of blockchain governance. We have offered insights into defining stakeholders, the distribution of bargaining power endogenous to the consensus mechanism, the role of exogenous governance structures, and the need to bootstrap networks.

While we have aimed to be descriptive here, our analysis has normative implications. Current on-chain governance models can only be partial because of the existence of implicit contracts that embed expectations of return among diverse stakeholders. Alternatively put, governance can be on-chain to the extent that control rights can be made explicit. Implicit contracts are unavoidable in public blockchains, given the open, repeated interactions between participants in the $n$-sided market and technology ecosystem and the entrepreneurial needs of network bootstrapping. Protocols concerned with blockchain governance ought to frame their thinking around the need to recognise the coordinating of consensus around the existence and persistence of these implicit contracts.

These considerations raise a further research agenda on blockchain governance. The blockchain industry lacks an extensive understanding of governance that corporate governance relies upon, and which in turn informs regulatory policy. Yet regulatory dilemmas around whether tokens represent ownership in a network (that is, are tokens shares) or where control over a network is vested (which speaks to the OECD's [88] concern with tacit collusion on blockchain networks) are already on-going. The proposal by Pierce [89] for a regulatory safe harbour that allows a bootstrapped network to be decentralised will pivot on better understanding than we have now of what constitutes decentralisation of control. A deeper understanding of how the interaction between bootstrapping and decentralised consensus has evolved will offer a guide for blockchain developers who seek to achieve long-run decentralisation.

\section{Competing interests:}

None declared.

Ethical approval:

Not applicable.

Author's contribution:

$D A$ and $C B$ designed and wrote the manuscript jointly and in its entirety

Funding:

None declared.

Acknowledgements:

The authors would like to acknowledge participants at the Wharton Cryptogovernance Workshop (July 2019) and the British Blockechain Association's International Scientific Conference (March 2020).

\section{References:}

[1] P. De Filippi and B. Loveluck, "The invisible politics of bitcoin: governance crisis of a decentralized infrastructure," Internet Policy Review, vol. 5, no. 3, 2016.

[2] P. G. Klein, J. T. Mahoney, A. M. McGahan, and C. N. Pitelis, "Organizational governance adaptation: Who is in, who is out, and who gets what," Academy of Management Review, vol. 44, no. 1, pp. 6-27, 2019.

[3] O. E. Williamson, The Mechanisms of Governance. Oxford University Press, 1999. 
[4] O. E. Williamson, "The institutions of governance," The American Economic Review, vol. 88, no. 2, pp. 75-79, 1998.

[5] D. C. North, "Institutions," Journal of Economic Perspectives, vol. 5, no. 1, pp. 97-112, 1991.

[6] O. E. Williamson, "The theory of the firm as governance structure: from choice to contract," Journal of Economic Perspectives, vol. 16, no. 3, pp. 171-195, 2002.

[7] K. Yeung and D. Galindo, "Why do public blockchains need formal and effective internal governance mechanisms?," European Journal of Risk Regulation, vol. 10, no. 2, pp. 359-375, 2019.

[8] E. Alston, "Constitutions and Blockchains: Competitive Governance of Fundamental Rule Sets," Center for Growth and Opportunity at Utah State University Working Paper Series, vol. 3, 2019.

[9] A. Narayanan, J. Bonneau, E. Felten, A. Miller, and S. Goldfeder, Bitcoin and Cryptocurrency Technologies: $A$ Comprehensive Introduction. Princeton University Press, 2016.

[10] Y.-Y. Hsieh, J.-P. Vergne, and S. Wang, "The internal and external governance of blockchain-based organizations: Evidence from cryptocurrencies," in Bitcoin and Beyond: Blockchains and Global Governance, vol. 48-68, M. Campbell-Verduyn, Ed. London and New York: Routledge, 2018.

[11] S. Nakamoto, "Bitcoin: A Peer-to-Peer Electronic Cash System,” 2008.

[12] R. Nyffenegger, "Scaling Bitcoin," Master's, Universität Basel, 2018.

[13] W. Reijers et al., "Now the code runs itself: On-chain and off-chain governance of blockchain technologies," Topoi, pp. 1-11, 2018.

[14] P. Honkanen, M. Westerlund, and M. Nylund, "Governance in Decentralized Ecosystems," CLOUD COMPUTING 2019, p. 59, 2019.

[15] L. Mosley, H. Pham, and Y. Bansal, "Towards a Systematic Understanding of Blockchain Governance in Proposal Voting: A Dash Case Study," Available at SSRN 3416564, 2019.

[16] M. Rauchs et al., "Distributed Ledger Technology Systems: A Conceptual Framework," SSRN, 2018.

[17] R. H. Coase, "The Nature of the Firm," Economica, vol. 4, no. 16, pp. 386-405, 1937.

[18] J. M. Buchanan and G. Tullock, The Calculus of Consent. Ann Arbor: University of Michigan Press 1962.

[19] J. M. Buchanan, "An Economic Theory of Clubs," Economica, vol. 32, no. 125, pp. 1-14, 1965.

[20] O. D. Hart, Firms, Contracts, and Financial Structure. Oxford and New York: Clarendon Press, 1995.

[21] O. D. Hart, "Incomplete Contracts and the Theory of the Firm," Journal of Law, Economics, \& Organization, vol. 4, no. 1, pp. 119-139, 1988.

[22] O. E. Williamson, The Economic Institutions of Capitalism. NY: Free Press, 1985.

[23] E. Ostrom, Governing the Commons: The Evolution of Institutions for Collective Action. Cambridge: Cambridge University Press, 1990.
[24] J. R. Commons, "Institutional economics," The American Economic Review, pp. 648-657, 1931.

[25] R. H. Coase, "The problem of social cost," The Journal of Law and Economics, vol. 56, no. 4, pp. 837-877, 1960.

[26] S. Davidson, P. De Filippi, and J. Potts, "Blockchains and the economic institutions of capitalism," Journal of Institutional Economics, vol. 14, no. 4, pp. 639-658, 2018.

[27] C. Berg, S. Davidson, and J. Potts, Understanding the Blockechain Economy: An Introduction to Institutional Cryptoeconomics. Edward Elgar Publishing, 2019.

[28] C. Catalini and J. S. Gans, "Some simple economics of the blockchain," National Bureau of Economic Research, 2016.

[29] D. W. E. Allen, C. Berg, B. Markey-Towler, M. Novak, and J. Potts, "Blockchain and the Evolution of Institutional Technologies: Implications for Innovation Policy," Research Policy, 7 August 2019.

[30] D. W. E. Allen, A. Berg, and B. Markey-Towler, "Blockchain and Supply Chains: V-Form Organisations, Value Redistributions, De-Commoditisation and Quality Proxies," The Journal of the British Blockechain Association, vol. 2, no. 1, pp. 57-65, 2019.

[31] W. W. Powell, "Neither Market Nor Hierarchy: Network forms of organization," Research in Organizational Behavior, vol. 12, pp. 295-336, 1990.

[32] J. Wareham, P. B. Fox, and J. L. Cano Giner, "Technology ecosystem governance," Organization science, vol. 25, no. 4, pp. 1195-1215, 2014.

[33] S. Burris, P. Drahos, and C. Shearing, "Nodal governance," Australian Journal of Legal Philosophy, vol. 30, p. 30, 2005.

[34] C. Berg, S. Davidson, and J. Potts, "Proof of work as a three-sided market," Frontiers in Blockchain, 31 January 2020.

[35] M. Friedman, "The social responsibility of business is to increase its profits," in New York Times Magazine, ed, 1970.

[36] A. A. Alchian and H. Demsetz, "Production, Information Costs, and Economic Organization," The American Economic Review, vol. 62, no. 5, pp. 777-795, 1972.

[37] A. Shleifer and R. W. Vishny, "A Survey of Corporate Governance," The Journal of Finance, vol. 52, no. 2, pp. 737-783, 1997.

[38] H. R. Bowen, Social Responsibilities of the Businessman. University of Iowa Press, 1953.

[39] T. Donaldson and L. E. Preston, "The stakeholder theory of the corporation: Concepts, evidence, and implications," Academy of management Review, vol. 20, no. 1, pp. 65-91, 1995.

[40] J. F. Vos, "Corporate social responsibility and the identification of stakeholders," Corporate Social Responsibility and Environmental Management, vol. 10, no. 3, pp. 141-152, 2003.

[41] R. E. Freeman, Strategic Management: A Stakeholder Approach. Pitman, 1984. 
[42] M. E. Clarkson, "A stakeholder framework for analyzing and evaluating corporate social performance," Academy of management review, vol. 20, no. 1, pp. 92-117, 1995.

[43] E. M. Dodd Jr, "For whom are corporate managers trustees," Harv. L. Rev., vol. 45, p. 1145, 1931.

[44] L. E. Preston and H. J. Sapienza, "Stakeholder management and corporate performance," Journal of behavioral Economics, vol. 19, no. 4, pp. 361-375, 1990.

[45] S. DiRose and M. Mansouri, "Comparison and Analysis of Governance Mechanisms Employed by BlockchainBased Distributed Autonomous Organizations," in 2018 13th Annual Conference on System of Systems Engineering (SoSE), 2018, pp. 195-202: IEEE.

[46] N. Carter, "A Cross-Sectional Overview of Cryptoasset Governance and Implications for Investors," 2016.

[47] M. J. Roe, "The shareholder wealth maximization norm and industrial organization," University of Pennsylvania Law Review, vol. 149, p. 2063, 2000.

[48] Y. Fassin, "A dynamic perspective in Freeman's stakeholder model," Journal of Business Ethics, vol. 96, no. 1, p. 39, 2010.

[49] Y. Fassin, "The stakeholder model refined," Journal of Business Ethics, vol. 84, no. 1, pp. 113-135, 2009.

[50] S. J. Grossman and O. D. Hart, "The costs and benefits of ownership: A theory of vertical and lateral integration," Journal of Political Economy, vol. 94, no. 4, pp. 691-719, 1986.

[51] P. G. Klein, J. T. Mahoney, A. M. McGahan, and C. N. Pitelis, "Who is in charge? A property rights perspective on stakeholder governance," Strategic Organization, vol. 10, no. 3, pp. 304-315, 2012.

[52] G. Baker, R. Gibbons, and K. J. Murphy, "Relational contracts and the theory of the firm," The Quarterly Journal of Economics, vol. 117, no. 1, pp. 39-84, 2002.

[53] C. I. Barnard, The Functions of the Executive. Harvard University Press, 1938.

[54] J. S. Harrison, D. A. Bosse, and R. A. Phillips, "Managing for stakeholders, stakeholder utility functions, and competitive advantage," Strategic management journal, vol. 31, no. 1, pp. 58-74, 2010.

[55] C. Eesley and M. J. Lenox, "Firm responses to secondary stakeholder action," Strategic Management Journal, vol. 27, no. 8, pp. 765-781, 2006.

[56] P. de Filippi and G. Mcmullen, "Governance of blockchain systems: Governance of and by Distributed Infrastructure," Blockchain Research Institute and COALA, 2018.

[57] J. Song, "Bitcoin, UASF and Skin in the Game," in Medium, ed, 2017.

[58] E. G. Sirer, "Time for Bitcoin Users to Reclaim Their Voice," ed, 2016.

[59] V. Buterin, "Governance, Part 2: Plutocracy Is Still Bad," in Vitalik Buterin's website, ed, 2018.

[60] V. Buterin, "Notes on Blockchain Governance," in Vitalik Buterin's website, ed, 2017.
V. Zamfir, "Against on-chain governance," in Medium, ed, 2017.

[62] F. Ehrsam, "Blockchain Governance: Programming Our Future," in Medium, ed, 2017.

[63] Digital Currency Group, "Bitcoin Scaling Agreement at Consensus 2017," 23 May 2017, Available: https://medium.com/@DCGco/bitcoin-scalingagreement-at-consensus-2017-133521fe9a77.

[64] A. Berg, C. Berg, and M. Novak, "Blockchains and constitutional catallaxy," Constitutional Political Economy, forthcoming.

[65] M. Prewitt and S. McKie, "Blockchain communities and their emergent governance," in Amentum blog, ed: Medium, 2018.

[66] I. M. Kirzner, Discovery and the Capitalist Process. University of Chicago Press, 1985.

[67] D. W. E. Allen, “The Private Governance of Entrepreneurship: An Institutional Approach to Entrepreneurial Discovery," PhD (Economics), School of Economics, Finance and Marketing, RMIT University, Melbourne, 2017.

[68] J. Potts, Innovation Commons: The Origin of Economic Growth. Oxford University Press, 2019.

[69] K. Dopfer and J. Potts, The general theory of economic evolution. Routledge, 2015.

[70] Y. Chen, "Blockchain tokens and the potential democratization of entrepreneurship and innovation," Business horizons, vol. 61, no. 4, pp. 567-575, 2018.

[71] J. M. Woodside, F. K. Augustine Jr, and W. Giberson, "Blockchain technology adoption status and strategies," Journal of International Technology and Information Management, vol. 26, no. 2, pp. 65-93, 2017.

[72] D. W. E. Allen, "Governing the Entrepreneurial Discovery of Blockchain Applications," Journal of Entrepreneurship and Public Policy, forthcoming.

[73] J. Potts, "Hype as a public good for innovation," Available at SSRN 2934675, 2017.

[74] J. Assange, When Google Met Wikileaks. OR Books, 2016.

[75] S. Nakamoto. (2010). Re: Wikileaks contact info? Available: https:/ / bitcointalk.org/index.php?topic $=1735 . \mathrm{msg} 2699$ 9\#msg26999

[76] D. M. Kreps, "Corporate culture and economic theory," in Firms, Organizations and Contracts, Oxford University Press, Oxford, J. E. Alt and K. A. Shepsle, Eds., 1996, pp. 221275.

[77] J. Jeffers and M. Lee, "Corporate Culture as an Implicit Contract," presented at the ASSA Annual Meeting, Atlanta, Georgia, 5 January 2019. Available: https://www.aeaweb.org/conference/2019/preliminary /902?q=eNqrVipOLS7OzM8LqSxIVbKqhnGVrJQMl Wp11BKLi_OTgRwlHaWS1KJcXAirLDO1HKQ2JbUk MTNcJzUFrFhHqaigXDDEMlwwAZDelMRKqM7M3 FQQqxZcMIhcliBv

[78] C. Camerer and A. Vepsalainen, "The economic efficiency of corporate culture," Strategic Management Journal, vol. 9, no. S1, pp. 115-126, 1988. 
[79] P. A. French, "The corporation as a moral person," American Philosophical Quarterly, vol. 16, no. 3, pp. 207215, 1979.

[80] W. G. Weaver, "Corporations as intentional systems," Journal of Business Ethics, vol. 17, no. 1, pp. 87-97, 1998.

[81] M. C. Jensen and W. H. Meckling, "Theory of the firm: Managerial behavior, agency costs and ownership structure," Journal of financial economics, vol. 3, no. 4, pp. 305-360, 1976.

[82] G. G. Sollars, “The corporation: Genesis, identity, agency," in The Routledge Companion to Business Ethics: Routledge, 2018, pp. 239-256.

[83] C. Berg, S. Davidson, and J. Potts, "Blockchain technology as economic infrastructure: Revisiting the electronic markets hypothesis," Frontiers in Blockechain, vol. 2, p. 22, 2019.

[84] B. M. Frischmann, Infrastructure: The Social V alue of Shared Resources. Oxford University Press, USA, 2012.

[85] United Nations Working Group on Internet

Governance, "Report of the Working Group on Internet Governance," United Nations, June 2005, Available: http://www.wgig.org/docs/

WGIGREPORT.pdf.

[86] M. J. Van Eeten and M. Mueller, "Where is the governance in Internet governance?," New media \& society, vol. 15, no. 5, pp. 720-736, 2013.

[87] M. Mueller, Will the Internet Fragment?: Sovereignty, Globalization and Cyberspace. Wiley, 2017.

[88] Organisation for Economic Co-operation and Development, "Blockchain Technology and Competition Policy - Issues paper by the Secretariat," 8 June 2018, Available: https:/ / one.oecd.org/document/ DAF/COMP/WD(2018)47/en/pdf.

[89] H. M. Pierce, "Running on Empty: A Proposal to Fill the Gap Between Regulation and Decentralization," Chicago, Illinois, 2020. 